\title{
On Achievable Error Exponents for Watermarking
}

\author{
Pierre Moulin and Ying Wang \\ Beckman Institute, Coordinate Science Lab \\ and Department of Electrical and Computer Engineering \\ University of Illinois at Urbana-Champaign \\ Urbana, IL 61801, USA
}

\begin{abstract}
Probability-of-error exponents have recently been derived for watermarking systems based on spread-spectrum and quantization-index modulation methods. This paper takes this work one step further and presents minmax error exponents for any embedding scheme and any attack (subject to distortion constraints) at all rates below capacity. The decoders used are universal: they do not know the attack used. Randomized codes outperform deterministic codes, except in the case of memoryless attacks where the same performance is obtained using either kind of code.
\end{abstract}

Keywords: Watermarking, coding, universal decoding, probability of error, randomized codes, game theory.

\section{INTRODUCTION}

Recent years have seen increased activity in the information-theoretic aspects of watermarking, data hiding, and steganography. This requires a precise definition of the watermarking problem, with admissible strategies for the watermark embedder, adversary, and decoder. This motivated the game-theoretic approach of Moulin and O'Sullivan, ${ }^{1}$ where the worst attack channel $p(y \mid x)$ in a class of memoryless channels was derived, and capacity is the solution to a maxmin mutual-information game. This approach has been extended to the case of channels with arbitrary memory by Cohen and Lapidoth ${ }^{2}$ and Somekh-Baruch and Merhav. ${ }^{3,4}$ In these problems, the host source $S$ is memoryless, and capacity takes the form

$$
C=\sup _{p_{X U \mid S}} \min _{p_{Y \mid X}}[I(U ; Y)-I(U ; S)]
$$

where $U$ is an auxiliary random variable, and the sup and min are subject to appropriate constraints. This capacity formula is an extension of the formula derived by Gel'fand and Pinsker ${ }^{5}$ for channel coding with side information at the encoder.

The aforementioned model ${ }^{2-4}$ for attack channels with arbitrary memory is reminiscent of the classical memoryless arbitrary varying channel $(\mathrm{AVC})^{6-8}$ which is often used to analyze jamming problems. In the classical AVC model, no side information is available to the encoder or decoder.

In many applications, one does not require transmission of hidden data at a rate approaching capacity. Rather one is interested in minimizing error probability at a transmission rate of interest, which could be well below capacity. This type of analysis has attracted attention recently and has been used to optimize parameters of spread-spectrum and quantization-index-modulation systems, see e.g.,. ${ }^{9-12}$

While exact analysis of the error probability is generally infeasible, analysis of the exponential rate of decay of this probability (or error exponent) is more tractable. There is a vast body of information-theoretic literature describing optimal error exponents for point-to-point communication, ${ }^{6,8}$ but very little is available on problems of communication with side information (such as watermarking). Recent work in this direction includes private watermarking, ${ }^{3}$ in which the host signal is known to both the encoder and the decoder. Problems such as public watermarking are more complex. In this case, capacity is achievable by nontraditional means, e.g. binning

E-mails: moulin@ifp.uiuc.edu, ywang@ifp.uiuc.edu. 


\section{Host Sequence}

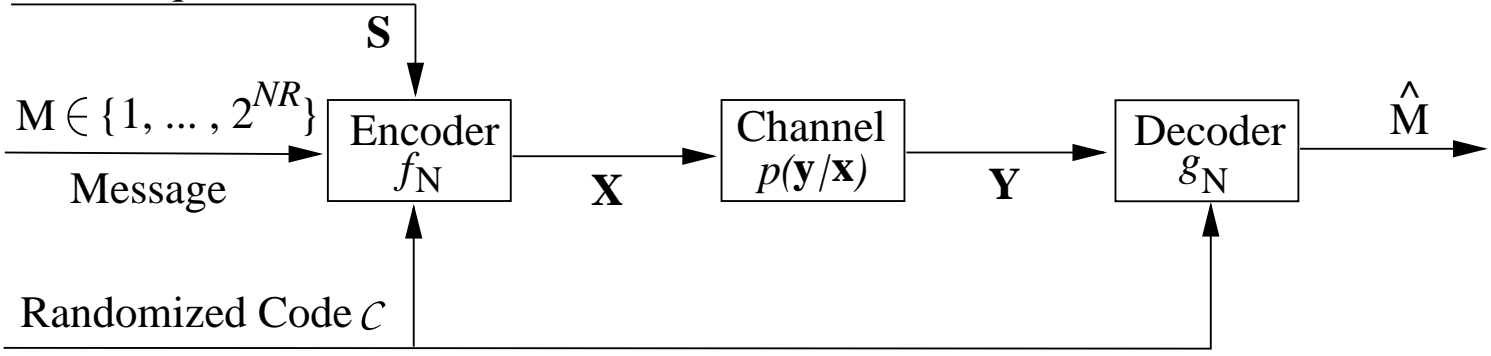

Figure 1. The public watermarking problem. The encoder and attack channel are subject to distortion constraints.

schemes. We show that optimal error exponents are achievable by binning schemes as well, albeit different from capacity-achieving binning schemes. *

All our derivations are based on the method of types ${ }^{6}$ and are presented in detail in our preprint ${ }^{13}$ for this problem and a more general one where encoder, adversary and decoder have access to degraded versions of a channel state sequence. The case where the probability mass function (p.m.f.) of the host sequence is unknown can also be accommodated in our framework.

Throughout, we use uppercase letters for random variables, lowercase letters for individual values, and boldface fonts for sequences. The symbols $D(p \| q)=\sum_{x} p(x) \log _{2} \frac{p(x)}{q(x)}$ and $I(X ; Y)=\sum_{x, y} p(x) p(y \mid x) \log _{2} \frac{p(y \mid x)}{p(y)}$ denote the Kullback-Leibler divergence between two p.m.f.'s $p$ and $q$ and the mutual information between two random variables $X$ and $Y$, respectively; the latter will be frequently denoted by $\tilde{I}_{X ; Y}\left(p_{X Y}\right)$ to make the dependency on the joint p.m.f. $p_{X Y}$ explicit.

Following the notation in Csiszár and Körner, ${ }^{6}$ let $p_{\mathbf{x}}$ denote the type of a sequence $\mathbf{x} \in \mathcal{X}^{N}$, i.e., the frequency of occurrence of each element of $\mathcal{X}$ in the sequence $\mathbf{x}$. A type is an empirical p.m.f. over $\mathcal{X}$. For instance, if $\mathcal{X}=\{0,1\}, N=5$ and $\mathbf{x}=(0,1,1,0,1)$, we obtain $p_{\mathbf{x}}=\left(\frac{2}{5}, \frac{3}{5}\right)$. Likewise, we define the joint type $p_{\mathbf{x y}}$ of a pair of sequences $(\mathbf{x}, \mathbf{y}) \in \mathcal{X}^{N} \times \mathcal{Y}^{N}$, which is an empirical p.m.f. over $\mathcal{X} \times \mathcal{Y}$. Finally, we define the conditional type $p_{\mathbf{y} \mid \mathbf{x}}$ of a pair of sequences $(\mathbf{x}, \mathbf{y})$ as $\frac{p_{\mathbf{x y}}(x, y)}{p_{\mathbf{x}}(x)}$ for all $x \in \mathcal{X}$ such that $p_{\mathbf{x}}(x)>0$.

\section{STATEMENT OF THE PROBLEM}

Our public watermark communication problem is diagrammed in Fig. 1. The host sequence $\mathbf{S}=\left(S_{1}, \ldots, S_{N}\right)$ consists of independent and identically distributed (i.i.d.) samples drawn from a p.m.f. $p_{S}(s), s \in \mathcal{S}$. This sequence is available to the encoder but not to the adversary and decoder. The adversary's channel is of the form $p_{\mathbf{Y} \mid \mathbf{X}}(\mathbf{y} \mid \mathbf{x})$.

A message $M$ is to be transmitted to a decoder; $M$ is uniformly distributed over the message set $\mathcal{M}$. The transmitter produces a sequence $\mathbf{X}=f_{N}(\mathbf{S}, M)$. The adversary passes $\mathbf{X}$ through the channel $p_{\mathbf{Y} \mid \mathbf{X}}(\mathbf{y} \mid \mathbf{x})$ to produce corrupted data $\mathbf{Y}$. The decoder does not know $p_{\mathbf{Y} \mid \mathbf{X}}$ selected by the adversary and produces an estimate $\hat{M}=g_{N}(\mathbf{Y}) \in \mathcal{M}$ of the transmitted message. The alphabets for $X$ and $Y$ are denoted by $\mathcal{X}$ and $\mathcal{Y}$, respectively. We allow the encoder/decoder pair $\left(f_{N}, g_{N}\right)$ to be randomized, i.e., the choice of $\left(f_{N}, g_{N}\right)$ is a function of a random variable known to the encoder and decoder but not to the adversary. This random variable is independent of all other random variables and plays the role of a secret key. The randomized code will be denoted by $\left(F_{N}, G_{N}\right)$.

\footnotetext{
${ }^{*}$ Similarly to conventional problems of transmission without side information, ${ }^{6}$ capacity-achieving codes are generally suboptimal in terms of error exponents at rates below capacity.
} 


\subsection{Constrained Side-Information Codes}

A distortion function $d(s, x)$ is defined to quantify embedding distortion; the same function is used to quantify the distortion due to the attack. The definition is extended to $N$-vectors using $d^{N}(\mathbf{s}, \mathbf{x})=\frac{1}{N} \sum_{i=1}^{N} d\left(s_{i}, x_{i}\right)$.

We now define a class of codes satisfying maximum-distortion constraints (Def. 2.1) and a class of codes satisfying average-distortion constraints (Def. 2.2). The latter class is of course larger than the former. Def. 2.2 is analogous to the definition of a length- $N$ information hiding code. ${ }^{1}$ The common source of randomness between encoder and decoder appears via the distribution $p\left(f_{N}, g_{N}\right)$ whereas in the paper ${ }^{1}$ it appears via a cryptographic key sequence $\mathbf{k}$ with finite entropy rate. We also define a class of randomly-modulated (RM) codes (Def. 2.3), which is a special class of randomized codes. The RM terminology is adopted from Hughes and Thomas. ${ }^{14}$

DEFINITION 2.1. A length- $N$, rate- $R$, randomized code with side information and maximum distortion $D_{1}$ is a triple $\left(\mathcal{M}, F_{N}, G_{N}\right)$, where

- $\mathcal{M}$ is the message set of cardinality $|\mathcal{M}|=\left\lceil 2^{N R}\right\rceil$;

- $\left(F_{N}, G_{N}\right)$ has joint distribution $p\left(f_{N}, g_{N}\right)$;

- $f_{N}: \mathcal{S}^{N} \times \mathcal{M} \rightarrow \mathcal{X}^{N}$ is the encoder mapping the host sequence $\mathbf{s}$ and message $m$ to the transmitted sequence $\mathbf{x}=f_{N}(\mathbf{s}, m)$. The mapping is subject to the distortion constraint

$$
d^{N}\left(\mathbf{s}, f_{N}(\mathbf{s}, m)\right) \leq D_{1} \quad \text { almost surely }\left(p_{S} p_{F_{N}} p_{M}\right) ;
$$

- $g_{N}: \mathcal{Y}^{N} \rightarrow \mathcal{M}$ is the decoder mapping the received sequence $\mathbf{y}$ to a decoded message $\hat{m}=g_{N}(\mathbf{y})$.

Definition 2.2. A length- $N$, rate- $R$, randomized code with side information and expected distortion $D_{1}$ is a triple $\left(\mathcal{M}, F_{N}, G_{N}\right)$ which satisfies the same conditions as in Def. 2.1, except that (1) is replaced with the weaker constraint

$$
\sum_{\mathbf{s}} p(\mathbf{s}) \sum_{f_{N}} p\left(f_{N}\right) \sum_{m \in \mathcal{M}} \frac{1}{|\mathcal{M}|} d^{N}\left(\mathbf{s}, f_{N}(\mathbf{s}, m)\right) \leq D_{1}
$$

DEFINITION 2.3. A randomly modulated (RM) code with side information is a randomized code defined via permutations of a prototype $\left(f_{N}, g_{N}\right)$. Such codes are of the form

$$
\begin{aligned}
\mathbf{x}=f_{N}^{\pi}(\mathbf{s}, m) & \triangleq \pi^{-1} f_{N}(\pi \mathbf{s}, m) \\
g_{N}^{\pi}(\mathbf{y}) & \triangleq g_{N}(\pi \mathbf{y})
\end{aligned}
$$

where $\pi$ is chosen uniformly from the set of all $N$ ! permutations, and the sequence $\pi \mathbf{x}$ is obtained by applying $\pi$ to the elements of $\mathbf{x}$.

\subsection{Constrained Attack Channels}

Next we define a class $\mathcal{A}$ of discrete memoryless channels (C-DMC - Def. 2.4) and a closely related class $\mathcal{P}_{\mathbf{Y} \mid \mathbf{X}}[\mathcal{A}]$ of AVC's (Def. 2.5).

Definition 2.4. The compound DMC (C-DMC) class $\mathcal{A}$ is a subset of $\mathcal{P}_{Y \mid X}$. The set $\mathcal{A}$ is defined according to the application. For instance $\mathcal{A}$ could be defined as the class of DMC's that introduce expected distortion at most equal to $D_{2}$ :

$$
\sum_{x, y} p_{X}(x) p_{Y \mid X}(y \mid x) d(x, y) \leq D_{2} .
$$

$\mathcal{A}$ can also be defined to be a subset of the above class. In the case of a known channel, ${ }^{5} \mathcal{A}$ is a singleton.

DEFINITION 2.5. The AVC class $\mathcal{P}_{\mathbf{Y} \mid \mathbf{X}}[\mathcal{A}]$ is the set of channels such that for any channel input $\mathbf{x}$ and output $\mathbf{y}$, the empirical conditional p.m.f. (conditional type) $p_{\mathbf{y} \mid \mathbf{x}}$ belongs to $\mathcal{A}$ with probability 1:

$$
\operatorname{Pr}\left[p_{\mathbf{y} \mid \mathbf{x}} \in \mathcal{A}\right]=1 .
$$


If $\mathcal{A}$ is defined via the distortion constraint (3), condition (4) may be rewritten as

$$
\operatorname{Pr}\left[d^{N}(\mathbf{x}, \mathbf{y}) \leq D_{2}\right]=0,
$$

i.e., feasible channels have total distortion bounded by $N D_{2}$ and arbitrary memory.

\subsection{Probability of Error}

Denote by

$$
P_{e, N}=\min _{p\left(f_{N}, g_{N}\right)} \max _{p_{\mathbf{Y} \mid \mathbf{X}}} P_{e}\left(F_{N}, G_{N}, p_{\mathbf{Y} \mid \mathbf{X}}\right)
$$

the minmax probability of error. A rate $R$ is said to be achievable if $P_{e, N} \rightarrow 0$ as $N \rightarrow \infty$. The capacity $C\left(D_{1}, \mathcal{A}\right)$ is the supremum of all achievable rates. The minmax error exponent, or reliability function of the class of attack channels considered is defined as

$$
E(R) \triangleq \limsup _{N \rightarrow \infty}\left[-\frac{1}{N} \log P_{e, N}\right] .
$$

There are four combinations of maximum/expected distortion constraints for the transmitter and C-DMC/AVC designs for the adversary, and a question is whether same capacity and error exponents will be obtained in all four cases. We define a covert channel $p_{X U \mid S}$ as any channel such that

$$
\sum_{u, s, x} p_{X U \mid S}(x, u \mid s) p_{S}(s) d(s, x) \leq D_{1}
$$

where $U$ is any auxiliary random variable. We denote by $\mathcal{P}_{X U \mid S}\left(D_{1}\right)$ the set of covert channels.

Consider a quatuor of random variables $(S, U, X, Y)$ with joint p.m.f. $p_{S U X Y}$. The following difference of mutual informations plays a fundamental role in capacity analyses ${ }^{1,4}$ of public watermarking. It plays a central role in the analysis of error exponents as well:

$$
J\left(p_{S U X Y}\right) \triangleq I(U ; Y)-I(U ; S) .
$$

Capacity is the same under all four combinations of constraints $^{1,4}$ :

$$
C\left(D_{1}, \mathcal{A}\right)=\sup _{p_{X U \mid S} \in \mathcal{P}_{X U \mid S}\left(D_{1}\right)} \min _{p_{Y \mid X} \in \mathcal{A}} J\left(p_{S} p_{X U \mid S} p_{Y \mid X}\right) .
$$

\section{MAIN RESULTS}

In this section, we derive lower and upper bounds on the reliability function $E(R)$ of $(6)$. A lower bound on $E(R)$ is obtained by constructing a random codebook and proving the existence of a code that achieves this lower bound. As is typical in communication problems, such codes are very good at high rates but not necessarily so at low rates. The upper bound on $E(R)$ is proved using a sphere-packing technique. In all formulas below, the cardinality of the alphabet for $U$ is unbounded.

Theorem 3.1. For the C-DMC case (Def. 2.4) with maximum-distortion constraint (1) or expected-distortion constraint (2) on the transmitter, the reliability function is lower-bounded by the random-coding error exponent

$$
\begin{aligned}
E_{r}^{C-D M C}(R)= & \min _{\tilde{p}_{S}} \sup _{p_{X U \mid S} \in \mathcal{P}_{X U \mid S}\left(D_{1}\right)} \min _{\tilde{p}_{Y \mid X U S}} \min _{p_{Y \mid X} \in \mathcal{A}} \\
& \quad\left[D\left(\tilde{p}_{S} p_{X U \mid S} \tilde{p}_{Y \mid X U S}|| p_{S} p_{X U \mid S} p_{Y \mid X}\right)+\left|J\left(\tilde{p}_{S} p_{X U \mid S} \tilde{p}_{Y \mid X U S}\right)-R\right|^{+}\right] .
\end{aligned}
$$

Moreover, $E_{r}^{C-D M C}(R)=0$ if and only if $R \geq C$. 
TheOREm 3.2. For the AVC case (Def. 2.5) with maximum-distortion constraint (1) or expected-distortion constraint (2) on the transmitter, the reliability function is lower-bounded by the random-coding error exponent

$$
\begin{aligned}
& E_{r}^{A V C}(R)=\min _{\tilde{p}_{S}} \sup _{p_{X U \mid S} \in \mathcal{P}_{X U \mid S}\left(D_{1}\right)} \tilde{p}_{Y \mid X U S} \in \mathcal{P}_{Y \mid X U S}[\mathcal{A}] \\
& \quad\left[D\left(\tilde{p}_{S}|| p_{S}\right)+\tilde{I}_{Y ; U S \mid X}\left(\tilde{p}_{S} p_{X U \mid S} \tilde{p}_{Y \mid X U S}\right)+\left|J\left(\tilde{p}_{S} p_{X U \mid S} \tilde{p}_{Y \mid X U S}\right)-R\right|^{+}\right] .
\end{aligned}
$$

Moreover, $E_{r}^{A V C}(R)=0$ if and only if $R \geq C$.

The random-coding error exponents (9) and (10) are achieved by conditionally constant composition codes using two novel techniques: a stacked binning encoder and a maximum penalized mutual information decoder. In the C-DMC case, (9) is achieved by a deterministic code. In the AVC case, (10) is achieved using a randomly modulated code.

TheOREM 3.3. For the C-DMC case with maximum-distortion constraint (1) or expected-distortion constraint (2) on the transmitter, the reliability function is upper-bounded by the sphere-packing exponent

$$
E_{s p}^{C-D M C}(R)=\min _{\tilde{p}_{S}} \sup _{p_{X U \mid S} \in \mathcal{P}_{X U \mid S}\left(D_{1}\right)} \min _{\substack{\tilde{p}_{Y \mid X U S}: \\ J\left(\tilde{p}_{S} p_{X U \mid S} \tilde{p}_{Y \mid X U S}\right) \leq R}} \min _{p_{Y \mid X} \in \mathcal{A}} D\left(\tilde{p}_{S} p_{X U \mid S} \tilde{p}_{Y \mid X U S}|| p_{S} p_{X U \mid S} p_{Y \mid X}\right)
$$

for all $R_{\infty} \leq R \leq C$, where $R_{\infty}$ is the infimum of all $R$ such that $E_{s p}(R)<\infty$ in (11).

THeOREm 3.4. For the AVC case with maximum-distortion constraint (1) or expected-distortion constraint (2) on the transmitter, the reliability function is upper-bounded by the sphere-packing exponent

$$
E_{s p}^{A V C}(R)=\min _{\tilde{p}_{S}} \sup _{p_{X U \mid S} \in \mathcal{P}_{X U \mid S}\left(D_{1}\right)} \min _{\substack{\tilde{p}_{Y \mid X U S}: \\ \tilde{p}_{Y \mid X} \in \mathcal{A} \\ J\left(\tilde{p}_{S} p_{X U \mid S} \tilde{p}_{Y \mid X U S}\right) \leq R}}\left[D\left(\tilde{p}_{S} \| p_{S}\right)+\tilde{I}_{Y ; U S \mid X}\left(\tilde{p}_{S} p_{X U \mid S} \tilde{p}_{Y \mid X U S}\right)\right]
$$

for all $R_{\infty} \leq R \leq C$, where $R_{\infty}$ is the infimum of all $R$ such that $E_{s p}(R)<\infty$ in (11).

Theorem 3.5 determines the reliability function for C-DMC and AVC at high rates.

Theorem 3.5. For both the C-DMC and the AVC cases, there exists a critical rate $R_{c r}<C$ such that

$$
E_{r}(R)= \begin{cases}E_{s p}(R) & : R \geq R_{c r} \\ E_{s p}\left(R_{c r}\right)+R_{c r}-R & : \text { else }\end{cases}
$$

and $E(R)=E_{s p}(R)$ for all $R \geq R_{c r}$.

REMARK 3.1. The exponent $E_{r}^{C-D M C}(R)$ cannot exceed $E_{r}^{A V C}(R)$ at any rate.

REMARK 3.2. An upper bound on the error exponents (9) and (10) can be obtained by fixing $\tilde{p}_{S}=p_{S}$. In particular, this results in the upper bound $E_{r}^{A V C}(R) \leq|C-R|^{+}$; equality is achieved if the minimizing $\tilde{p}_{S}$ in (10) is equal to $p_{S}$, and $\tilde{p}_{Y \mid X U S}=p_{Y \mid X}$.

\section{BINARY-HAMMING CASE}

To illustrate the results of Section 3, we consider the case where $\mathcal{S}=\{0,1\} ; \mathbf{S}$ is a Bernoulli sequence with

$$
p_{1} \triangleq \operatorname{Pr}[S=1]=1-\operatorname{Pr}[S=0] ;
$$

transmission is subject to the distortion constraint (1) in which $d$ is Hamming distance; and the adversary is subject to the expected-distortion constraint (3) or to the maximum-distortion constraint (5). In both cases the set $\mathcal{A}$ is given by (3). We study three cases: 
Case I: $p_{1}=\frac{1}{2}, S$ unavailable to decoder. This problem is analogous to the public watermarking problem. $1,15,16$

Case II: $p_{1}=\frac{1}{2}, S$ available to encoder and decoder. This is the private watermarking problem (C-DMC case) studied in the paper. ${ }^{1}$ The AVC version of this problem ${ }^{3}$ is closely related to a problem studied by Csiszár and Narayan ${ }^{7}$ and Hughes and Thomas. ${ }^{14}$

Case III: Degenerate host: $p_{1}=0$, i.e., $S=0$ with probability one. Unlike, ${ }^{7,14}$ the attacker's noise may depend on $X$.

In all three cases, we were able to derive some analytical results and to numerically evaluate error exponents. Capacity formulas for these problems are given below and illustrated in Fig. 2.

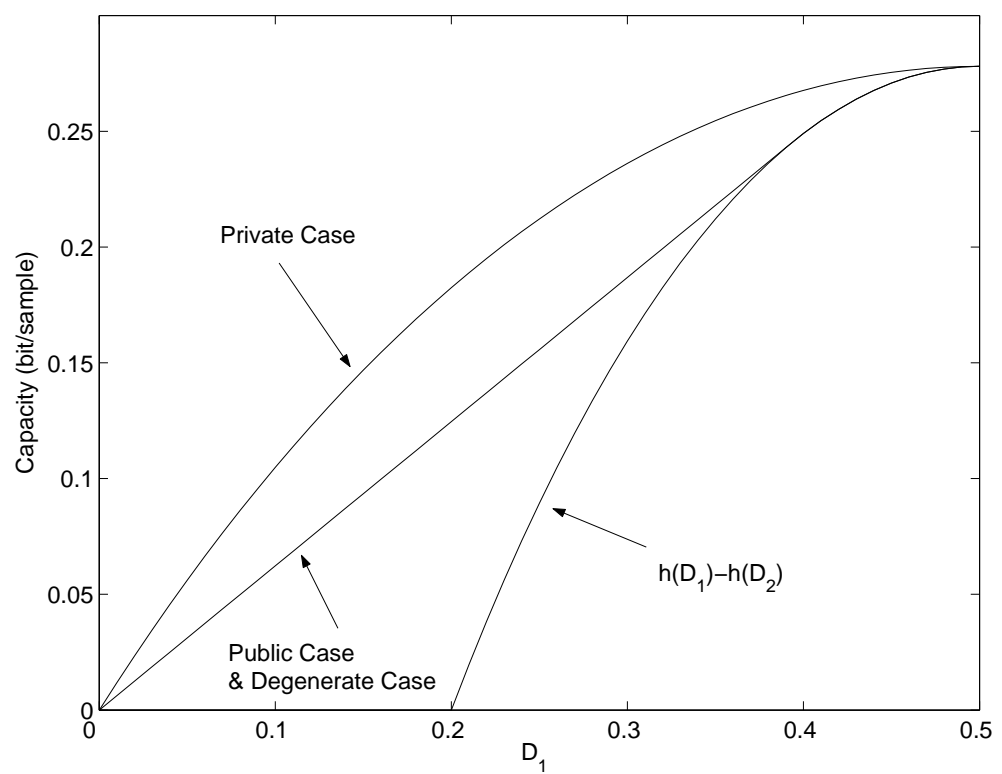

Figure 2. Capacity functions for Cases I-III when $D_{2}=0.2$.

\subsection{Case I: Public Watermarking}

Capacity for a fixed-DMC problem (adversary implements a binary symmetric channel (BSC) with crossover probability $D_{2}$ ) is given in Barron et al ${ }^{15}$ and Pradhan et al ${ }^{16}$ :

$$
C^{p u b}=g^{*}\left(D_{1}, D_{2}\right) \triangleq \begin{cases}\frac{D_{1}}{\delta_{2}}\left[h\left(\delta_{2}\right)-h\left(D_{2}\right)\right], & \text { if } 0 \leq D_{1}<\delta_{2} \\ h\left(\delta_{2}\right)-h\left(D_{2}\right), & \text { if } \delta_{2} \leq D_{1} \leq 1 / 2 \\ 1-h\left(D_{2}\right), & \text { if } D_{1}>1 / 2,\end{cases}
$$

where $\delta_{2}=1-2^{-h\left(D_{2}\right)}$ and $h(p)=-p \log _{2} p-(1-p) \log _{2}(1-p)$ is the binary entropy function. The straight-line portion of the capacity function is achieved by time-sharing. Proposition 4.1 shows that the BSC is the worst channel for the C-DMC and AVC classes considered.

Proposition 4.1. Capacity under the C-DMC and AVC models defined by the distortion constraints (3) and (5), respectively, is equal to $C^{p u b}$ and is achieved for $|\mathcal{U}|=2$.

Now let us turn our attention to error exponents. The sphere-packing exponent in the AVC case is trivial $(\infty)$ because its calculation requires minimization of a function over an empty set. The same phenomenon was observed and discussed in. ${ }^{14}$

Proposition 4.2. $E_{s p}^{A V C, p u b}(R)=\infty$ for all $R<C^{p u b}$. 
Proposition 4.3. The random-coding error exponent is a straight line in the AVC case:

$$
E_{r}^{A V C, p u b}(R)=\left|C^{p u b}-R\right|^{+}
$$

for all $R$. The minimizing $\tilde{p}_{S}$ in (10) coincides with $p_{S}$, the maximizing $|\mathcal{U}|=2$, and the minimizing $\tilde{p}_{Y \mid X U S}$ is the $B S C p_{Y \mid X}$ with crossover probability $D_{2}$.

Error exponents in the case $D_{1}=0.4, D_{2}=0.2$, are given in Fig. 3. For the C-DMC case, we have found numerically (see discussion in Sec. 4.4) that the worst attack channel $p_{Y \mid X}$ is the BSC with crossover probability $D_{2}$, and that the worst-case $\tilde{p}_{S}$ in (9) coincides with $p_{S}$.

\subsection{Case II: Private Watermarking}

Proposition 4.4.. ${ }^{1}$ Capacity is given by

$$
C^{\text {priv }}=h\left(D_{1} \star D_{2}\right)-h\left(D_{2}\right)
$$

where $p \star q \triangleq p(1-q)+(1-p) q$.

Proposition 4.5. $E_{s p}^{A V C, p r i v}(R)=\infty$ for all $R<C^{p u b}$. For $C^{p u b}<R \leq C^{\text {priv }}, E_{s p}^{A V C, p u b}(R)$ is achieved by $|\mathcal{U}|=2$.

Error exponents in the case $D_{1}=0.4, D_{2}=0.2$, are shown in Fig. 4 . As in Case I, for both the AVC and C-DMC cases, the worst-case $\tilde{p}_{S}$ in (9) and (10) coincides with $p_{S}$. In the AVC case, the critical rate $R_{c r}$ (see (13)) is very close to $C^{\text {priv }}$, and the worst attack channel $p_{Y \mid X}$ is the BSC with crossover probability $D_{2}$.

The capacity expression (15) was also derived for the AVC problem of Csiszár and Narayan, ${ }^{7}$ albeit with different assumptions. Error exponents for the latter problem were derived by Hughes and Thomas. ${ }^{14}$ They obtained $E_{r}^{A V C}(R)=|C-R|^{+}$and $E_{s p}^{A V C}(R)=\infty$ at all rates below capacity.

\subsection{Case III: Degenerate side information}

Proposition 4.6. Capacity is the same as in the public watermarking game: $C^{\text {deg }}=C^{\text {pub }}$.

Proposition 4.7. $E_{r}^{A V C, d e g}(R)=\left|C^{\text {deg }}-R\right|^{+}$for all $R<C^{\text {deg }}$. Proof: follows from Remark 3.2.

Proposition 4.8. $E_{s p}^{A V C, d e g}(R)=\infty$ for all $R<C^{d e g}$.

Unlike Case I and Case II, the worst attack is an asymmetric binary channel, favoring outputs with low Hamming weight. Error exponents in the case $D_{1}=0.4, D_{2}=0.2$, are given in Fig. 5 .

\subsection{Discussion}

In the numerical examples considered above, the gap between the random-coding and sphere-packing exponents in the C-DMC case is small. Comparing Figs. 3 and 4, we see that error exponents are larger in the private case (for both the C-DMC and AVC scenarios). This quantifies the loss in error exponent incurred by the decoder for having no side information available.

Some practical comments about the optimization problems solved in this section are in order. Among these problems, the calculations of random-coding and sphere-packing exponents for the C-DMC / public watermarking scenario are the most complicated ones, both of which have four layers of minimization or maximization. The number of the parameters to be optimized is $8|\mathcal{U}|+1\left(1\right.$ for $\tilde{p}_{S}, 4|\mathcal{U}|-2$ for $p_{X U \mid S}, 2$ for $p_{Y \mid X}$ and $4|\mathcal{U}|$ for $\left.\tilde{p}_{Y \mid X U S}\right)$. Other difficulties arise due to the lack of nice properties such as everywhere differentiability and convexity. There appears to be a substantial increase of difficulty going from $|\mathcal{U}|=2$ to larger $\mathcal{U}$. Based on the analytical results above, it is tempting to conjecture that $|\mathcal{U}|=2$ is an sufficient choice for optimality; unfortunately at this time we are unable to validate that conjecture analytically or numerically.

We have used a genetic algorithm ${ }^{17}$ to numerically solve the above-mentioned optimization problems. Advantages of genetic algorithms include easy implementation, robustness with respect to selection of starting points, no need for evaluation of function derivatives, and ability to handle high-dimensional problems. The parameters of a genetic algorithm may be selected to ensure that the algorithm is globally convergent. In particular, we have used an "elitist" genetic algorithm, in which the value of the best individual in each iteration is nondecreasing for a maximization problem (or nonincreasing for a minimization problem). The sequence of the best solutions in each iteration is guaranteed to converge to the global optimum almost surely. ${ }^{17,18}$ 


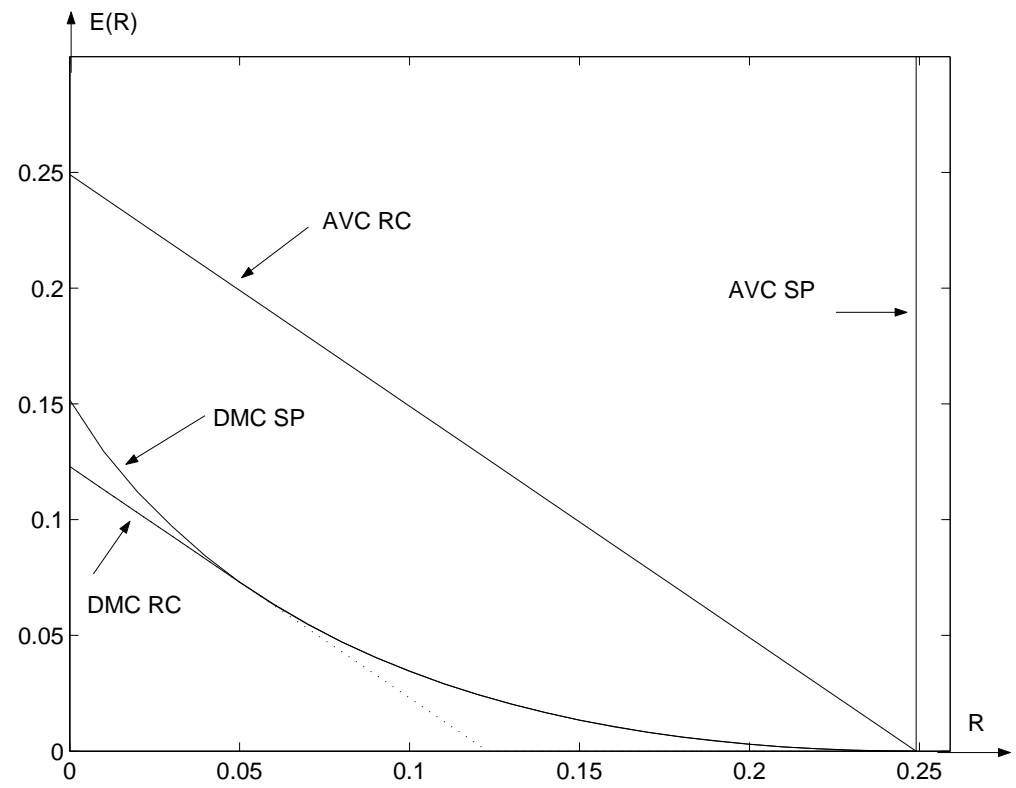

Figure 3. Error exponents for public watermarking $\left(D_{1}=0.4, D_{2}=0.2\right)$.

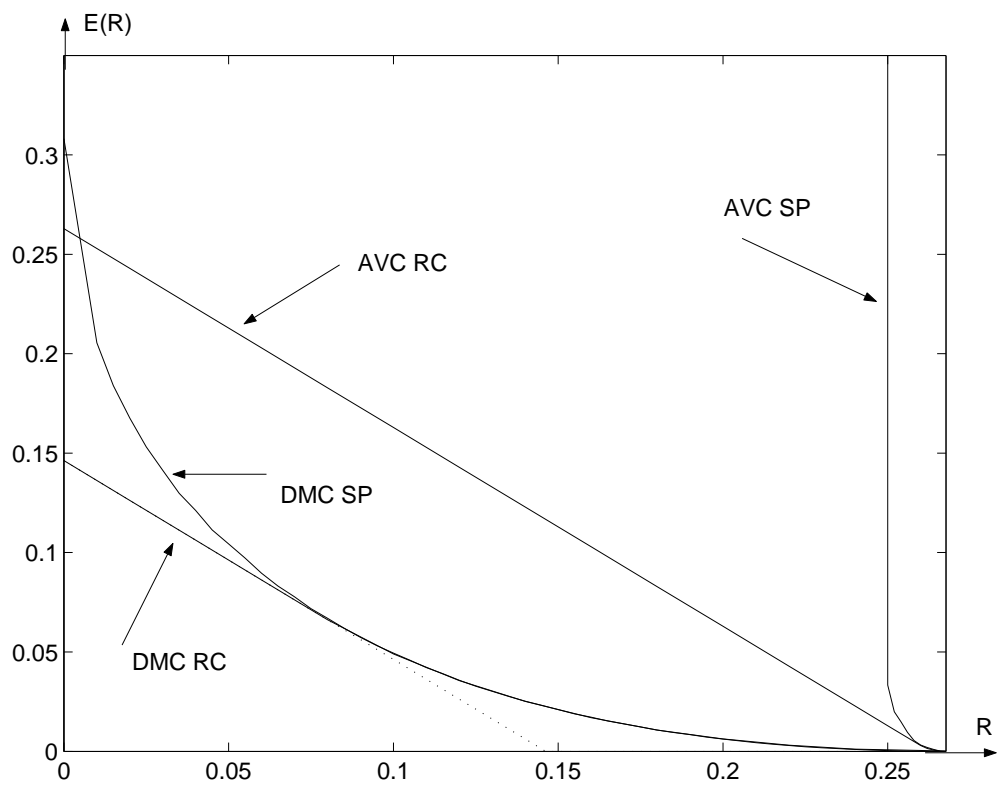

Figure 4. Error exponents for private watermarking $\left(D_{1}=0.4, D_{2}=0.2\right)$. 


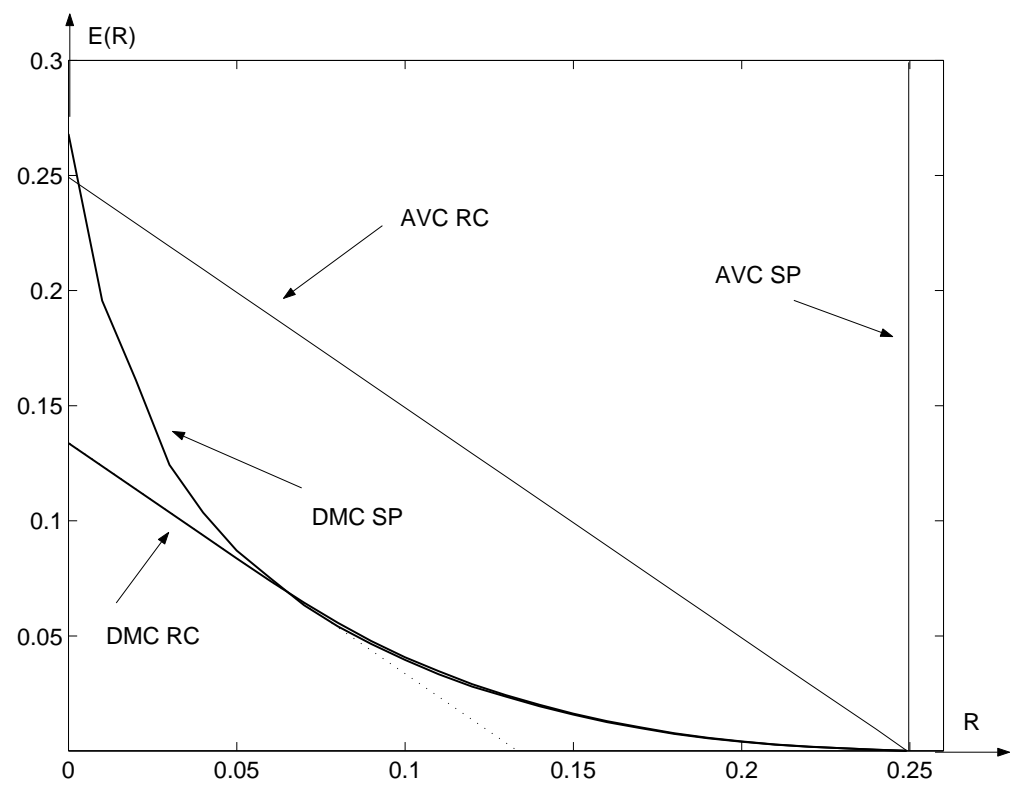

Figure 5. Error exponents for degenerate host $\left(D_{1}=0.4, D_{2}=0.2\right)$.

\section{ACKNOWLEDGMENTS}

This work was supported by NSF under ITR grants CCR 00-81268 and CCR 03-25924.

\section{REFERENCES}

1. P. Moulin and J. A. O'Sullivan, "Information-theoretic analysis of information hiding", IEEE Trans. on Information Theory, Vol. 49, No.3, pp. 563-593, March 2003.

2. A. S. Cohen and A. Lapidoth, "The Gaussian Watermarking Game," IEEE Trans. Information Theory, Vol. 48, No. 6, pp. 1639-1667, June 2002.

3. A. Somekh-Baruch and N. Merhav, "On the Error Exponent and Capacity Games of Private Watermarking Systems," IEEE Trans. on Information Theory, Vol. 49, No.3, pp. 537-562, March 2003.

4. A. Somekh-Baruch and N. Merhav, "On the Capacity Game of Public Watermarking Systems," IEEE Trans. on Information Theory, Vol. 50, No.3, pp. 511-524, March 2004.

5. S. I. Gel'fand and M. S. Pinsker, "Coding for Channel with Random Parameters," Problems of Control and Information Theory, Vol. 9, No. 1, pp. 19-31, 1980.

6. I. Csiszár and J. Körner, Information Theory: Coding Theory for Discrete Memoryless Systems, Academic Press, NY, 1981.

7. I. Csiszár and P. Narayan, "Arbitrarily Varying Channels with Constrained Inputs and States," IEEE Trans. Information Theory, Vol. 31, No. 1, pp. 42-48, Jan. 1988.

8. A. Lapidoth and P. Narayan, "Reliable Communication Under Channel Uncertainty," IEEE Trans. Information Theory, Vol. 44, No. 6, pp. 2148-2177, Oct. 1998.

9. P. Moulin and A. Ivanović, "The Zero-Rate Spread-Spectrum Watermarking Game," IEEE Transactions on Signal Processing, Vol. 51, No. 4, pp. 1098-1117, Apr. 2003.

10. P. Comesaña, F. Pérez-González and F. Balado, "Optimal strategies for spread-spectrum and quantizedprojection image data hiding games with BER payoffs," Proc. ICIP, pp. II.479-482, Barcelona, Spain, Sep. 2003.

11. A. K. Goteti and P. Moulin, "Two Private, Perceptual Data-Hiding Games," Proc. IEEE Int. Conf. Ac. Sp. and Sig. Proc. (ICASSP), Montreal, Canada, May 2004.

12. A. K. Goteti and P. Moulin, "QIM Watermarking Games," Proc. IEEE Int. Conf. Image Proc. (ICIP), Singapore, Oct. 2004. 
13. P. Moulin and Y. Wang, "Error Exponents for Channel Coding with Side Information," preprint, Sep 2004. www.ifp.uiuc.edu/ moulin/Papers/ErrExpSI04.ps. Short version in Proc. IEEE Information Theory Workshop, San Antonio, TX, Oct. 2004.

14. B. L. Hughes and T. G. Thomas, "On Error Exponents for Arbitrarily Varying Channels," IEEE Trans. on Information Theory, Vol. 42, No.1, pp. 87-98, Jan. 1996.

15. R. J. Barron, B. Chen, G. W. Wornell, "The duality between information embedding and source coding with side information and some applications", IEEE Trans. on Information Theory, Vol. 49, No. 5, pp. 1159-1180, May 2003.

16. S. S. Pradhan, J. Chou and Ramchandran, "Duality Between Source Coding and Channel Coding and its Extension to the Side Information Case," IEEE Trans. on Information Theory, Vol. 49, No. 5, pp. 1181-1203, May 2003.

17. Z. Michalewicz, Genetic Algorithms + Data Structures = Evolution Programs. New York: Springer, 1996.

18. G. Rudolph, Convergence Properties of Evolutionary Algorithms. Verlag Dr. Kovac: Hamburg, Germany, 1997. 